\title{
Mechanical Property and Strain Transferring Mechanism in Optical Fiber Sensors
}

\author{
Dongsheng Li, Liang Ren and Hongnan Li \\ Dalian University of Technology
}

China

\section{Introduction}

Optical fiber sensors (OFSs) have attracted considerable interests for their superior sensing abilities, especially due to their electromagnetic inference immunity and high sensitivity. However, OFS sensors based on bare fibers are fragile and easily damaged. For their safe use in engineering sensing, the glass core of optical fibers has to be coated with protective coatings, or to be bonded with adhesive materials, for instance, epoxy. Low elastic modulus of the soft coatings or bonding layer results in various shear stresses along the middle layer between the fiber core and the host structure to be measured. A portion of the host material strain is absorbed by the protective coatings when the strain transfers from the host material to the fiber core, and hence only a small fraction of structural strain is sensed. Consequently, the strains directly sensed by OFSs are different from actual structural strains. This is the main issue to be discussed in the chapter.

For the purpose to discuss the strain transferring problem, fundamental mechanical properties of OFSs are first presented. The chapter then develops an analytical model to derive the relationship between OFS sensed strains and actual structural strains. It is discovered that the strains, directly sensed by an OFS, have to be magnified by a factor (strain transfer rate) to reflect actual structural strains. This is, of course, of paramount interests for the application of optical fiber sensors. The factors that affect the efficiency of strain transfer on the optical fiber sensor are deduced and discussed in details based on the theoretical analysis. Critical adherence length is proposed as a measure to guarantee sufficient strain transfer rate based on the analytical model. Detailed analysis shows that the critical adherence length is the minimum length with which the fiber has to be tightly embedded to a structure for adequate sensing. Furthermore, theoretical results are verified through laboratory experimentation with the fiber Bragg grating sensors. Measured average strain transfer rates agree well with those calculated from the analytical model. Furthermore, the strain transfer rate of an OFS embedded in a multi-layered structure is consequently developed and the shear modulus of the host material on the influence of strain transmission is discussed.

For surface-bonded OFSs, optical fibers are adhered on the surfaces of structural members. Adhesive material still exists in-between and optical fibers are only partial buried. The strain readings of OFSs can be heavily affected by the thickness and mechanical properties of the adhesive and therefore it is important to determine how these factors affect the 
measurements. Numerical finite element simulations are reviewed for strain transferring mechanism of surface-bonded OFSs. In the end, the chapter summarizes current investigations on strain transferring mechanism of optical fiber sensors and practical packaging issues.

\section{Fundamental mechanical properties of optical fibers}

An optical fiber is a flexible, transparent fiber made of very pure glass (silica) not much wider than a human hair that acts as a waveguide, or "light pipe", to transmit light between the two ends of the fiber (Thyagarajan \& Ghatak, 2007). Optical fiber typically consists of a transparent core surrounded by a transparent cladding material with a lower index of refraction.

\begin{tabular}{|l|l|l|l|}
\hline \multicolumn{1}{|c|}{\begin{tabular}{c} 
Materials Properties \\
\multicolumn{1}{|c|}{$(1)$}
\end{tabular}} & \multicolumn{1}{|c|}{$\begin{array}{c}\text { Symbols } \\
(2)\end{array}$} & \multicolumn{1}{c|}{\begin{tabular}{c}
\multicolumn{1}{c|}{ Values } \\
$(3)$
\end{tabular}} & \multicolumn{1}{c|}{$\begin{array}{c}\text { Unit } \\
(4)\end{array}$} \\
\hline Young's modulus of silica fiber & $\mathrm{E}$ & $7.3 \times 10^{10}$ & $\mathrm{~Pa}$ \\
\hline Young's modulus of silicon coating & $\mathrm{E}$ & $2.55 \times 10^{6}$ & $\mathrm{~Pa}$ \\
\hline Poisson's ratio of silicon coating & $\mathrm{V}$ & $0.17 \sim 0.48$ & - \\
\hline Shear modulus of silicon coating & $\mathrm{G}$ & $8.5 \times 10^{5}$ & $\mathrm{~Pa}$ \\
\hline Strength of silica fiber & $\mathrm{\sigma}$ & $0.35 \sim 4.8 \times 10^{10}$ & $\mathrm{~Pa}$ \\
\hline Breaking strain of silica fiber & $\mathrm{E}$ & $\sim 10 \%$ & - \\
\hline Density & $\rho$ & 2200 & $\mathrm{Kgm}^{-3}$ \\
\hline Coefficient of thermal expansion & $f$ & $0.54 \times 10^{-6}$ & $\mathrm{~K}^{-1}$ \\
\hline
\end{tabular}

Table 1. Typical mechanical properties of the optical fiber (Fernando et al., 2002; Measures, 2001)

Optical fibers are drawn from a molten glass perform. Although fluorozirconate, fluoroaluminate, and chalcogenide glasses as well as crystalline materials like sapphire, are used for optical fibers, silica is the most common material, especially for optical fiber sensing applications. Optical fibers made of fused silica are perfectly elastic until their breaking point. They are brittle and do not yield as do metals when overstressed. For the interests of sensing applications, key mechanical properties of silica fiber are listed in Table 1. Due to variation of manufacturing processes, mechanical properties of optical fibers may fluctuate around the nominate values in Table 1.

Although the strength of silica fiber is in excess of $4.8 \mathrm{Gpa}(\sim 7 \%$ strain), the practical stress limit is much lower due to the presence of many microscopic flaws. Proof tests are then carried out by manufactures to guarantee a safe operating stress and a predictable life. Typically, optical fibers are proof tested to $0.35 \mathrm{Gpa}$, corresponding to about $0.5 \%$ strain (Measures, 2001). For most sensor applications, the strength of silica fiber is much higher than that of structural members to be measured. Fatigue tests showed that optical fibers have a long working life when its operating stress is a small fraction of the proof stress. For most long term sensing application, OFSs can survive and are adequate.

Furthermore, fiber Bragg gratings (FBG) are the most common sensing elements among OFSs. Their strength is of considerable interests. Experiments showed that the formation 
of FBGs with UV light leads to a substantial weakening of the optical fiber (Feced et al., 1997). Controversy exists. Varelas et al. (1997) have demonstrated that producing FBGs with a continuous wave caused only minimal reduction in the strength of the optical fiber. Further investigations are needed to study the effects of fabrication of FBGs on the fiber strength.

\section{Strain transferring mechanism for embedded OFSs}

Conventionally, the values measured by optical fiber sensors were assumed to be actual structural strains (Baldwin et al., 2002). In fact, the strain measured by an OFS is different from actual host structure strain because of the difference between the optical fiber core modulus and the modulus of the fiber coating or the adhesive. Such strain difference depends on detailed packing measures of OFSs. Generally, there are three methods to integrate OFSs with host structures in terms of packing strategies: (1) direct integration, in which OFSs are directly embedded in or surfaced bonded to a host structure; (2) sensorpackaging integration, by which OFSs are first fixed in a small tube or bonded on the surface of a plate, and then the tube or the plate is anchored in the host structure; (3) clamping integration, in which an OFS is gripped at two ends by a bracket fixed on the host structure. No matter how the OFSs are packaged and integrated, the fiber core is brittle and has to be protected by adhesives or a coating layer in most sensing applications to avoid fiber breakage and to ascertain its long term stability. However, such a protection results in inconsistency between the fiber strain and the structural strain. Such discrepancies are neglected in many applications of OFSs by simply assuming that the fiber strain is consistent with the host structural strain (Friebele et al., 1999; Udd, 1995). Such assumption gives acceptable measurement results for the OFSs with long gauge length, in which the peak host strain can be fully transferred into the fiber strain, but cannot provide good measurement strains for short gauge OFSs, for instance, FBG sensors, in which the effect of the bonded fiber length on strain transfer between the fiber and host structure is significant (Galiotis et al., 1984). It is, thus, of primary importance to have a detailed knowledge of the relationship between the host structural strain and the fiber strain in order to correctly interpret structural strain from the strains sensed in the fiber.

Since the Young's modulus of the fiber is typically much larger than that of the adhesive or the coating as shown in Table 1, the axial elastic displacements of the fiber and the host material are different. In order to measure the strain of host materials accurately, there is a need to develop the relationship between the strain sensed by an OFS and the actual strain of the host material. Many researchers all over the world have made great efforts in this field and obtained notable achievements. Pak (1992) analyzed the strain transfer of a coated optical fiber embedded in a host composite, which is strained by a far-field longitudinal shear stress parallel to the optical fiber. In this case, the maximum shear transfer occurs when the shear modulus of the coating is the geometric mean of the shear moduli of the fiber and the host material. Ansari \& Libo (1998) developed a simply model for evaluating the strain transfer percentage from the surrounding matrix to a length of optical fiber embedded in it under the assumption that the strain at the middle of the bonded fiber is equal to that of the host structure at the same position. Experiments were performed with a white-light fiber-optic interferometer to confirm the theory results. Li et al (2002) considered the coating as an ideal elasto-plastic material and deduced the strain transfer coefficients 
when the host material experiences tensile stress and compression stress respectively. Galiotis (1984) designed a polydiacetylene single fiber in an epoxy resin host material subjected to tensile strain along the fiber direction, and measured the strains at all points along the length of the fiber by the method of resonance Raman. They found that the axial strain in the fiber rises from a finite value at the end of the fiber to a fairly constant value at the central portion of the fiber, and that the axial strain at the midpoint of the fiber is lower than that applied to the host material, which can be approximately explained by the shearlag model of Cox (1952).

This chapter mainly concerns the study of a single optical fiber embedded in a finite host structure, which is subjected to uniform axial stress. To derive the relationship between the strain measured by an optical fiber and the actual one experienced by the structure, a more realistic hypothesis is proposed in this chapter.

\subsection{Theoretical approach}

An optical fiber usually consists of three layers: fiber core, cladding and coating. The core diameter of a single mode fiber is usually $9 \mu \mathrm{m}$, and the value for a multiple-mode fiber is $50 \mu \mathrm{m}$ (Fig.1). The coating, i.e., the outer layer of an optical fiber, often has an outer diameter of $250 \mu \mathrm{m}$ and an inner diameter of $125 \mu \mathrm{m}$. The coating is usually made of plastics.

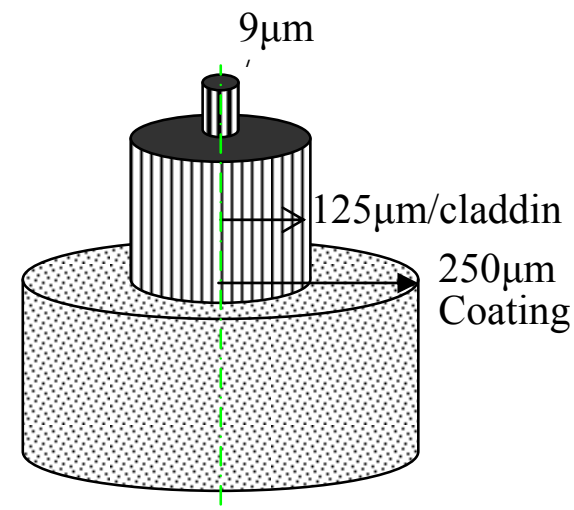

Fig. 1. Structure of a typical single mode fiber

We will deal with a concentric model of OFSs, which is similar to the fiber structure in Fig. 1. The concentric model is just a simplification of direct integration strategy. This model is suitable for two different cases. In the first case, a fiber with coating is directly embedded in the host structure. In the second case, the coating of a fiber is first stripped off, and then the bare fiber (including the core and cladding only) is attached to the inner surface of a steel tube by adhesives. In these two cases, there is an adhesive layer, often called middle layer, between the bare fiber and the host material. The three layers are concentric and the strain of the outer host material is transmitted to the inner fiber through the deformations of the middle layer.

For the concentric model, the strain transfer process from host material to the fiber core can be described as follows: as the host material generates strain under external loads, the 
deformation makes the shear strain appear in the interface between the host material and interlayer, and the shear strain is transferred to the interior of the interlayer to an extent which depends on the effective bonding of the interface. The interlayer generates axial strain at the same time. The axial strain in the interlayer is transferred to the fiber core through the shear strain in the interface between the interlayer and fiber core. The fiber core generates axial strain and senses the strain of the host material.

In the analysis of strain transfer to the optical fiber from the host material quantitatively, following assumptions are adopted for the concentric model.

Assumption 1) All the materials pertinent to the model remain elastic, and only the outer host material is subjected to axial stress and is uniformly strained, whereas the bare fiber and the middle layer do not directly bear any external loadings.

Assumption 2) Mechanical properties of the core and cladding of the fiber are the same. In reality, their properties are slightly different owing to their difference in some chemical components and the writing process of Bragg gratings. The core and cladding, hereinafter, are referred collectively as fiber for simplicity.

Assumption 3) There are no strain discontinuities across the interfaces, including the one between the host material and the middle layer and the one between middle layer and fiber interfaces, i.e. the bond between all the interfaces is perfect and no de-bonding exists.

In the concentric model, the Young's modulus of the single optical fiber is $\mathrm{E}_{g}$ with a radius of $r_{g}$ embedded in a host material, separated by a middle layer of Young's modulus $E_{c}$ and radius $r_{m}$ and Poisson's ratio $v$, as illustrated in Fig.2. The host material, assumed to be infinite in all directions, is subjected to a uniform axial stress. $L$ is the half gauge length of an optical fiber sensor, and $2 L$ is the total length that the fiber is bonded to the host material through the middle layer. $\tau(x, r)$ is the shear stress in the middle layer a distance $\mathrm{r}$ above a given $x$ coordinate along the center of the fiber. $\tau\left(x, r_{g}\right)$ is the shear stress at a given $x$ coordinate along the fiber-middle layer interface. $\sigma_{g}, \sigma_{c}$ and $\sigma_{m}$ are the axial stress in the fiber, middle layer and the host material, respectively.

Based on the stress equilibrium for a small segment of the fiber and assumptions 1-3, the longitudinal stress along the fiber $\sigma_{g}$, is related to the interfacial shear stress at the fiber/middle layer interface through,

$$
\frac{d \sigma_{g}}{d x}=-\frac{2 \tau\left(x, r_{g}\right)}{r_{g}}
$$

In reference to the free body diagram pertaining to the middle layer (Fig.2c), the equilibrium in the $x$ direction results in the following relationship by balancing the shear stresses $\tau\left(x, r_{g}\right)$ and $\tau(x, r)$ with the axial stress $\sigma_{c}$,

$$
\tau(x, r)=\frac{r_{g}}{r} \tau\left(x, r_{g}\right)-\frac{r^{2}-r_{g}{ }^{2}}{2 r} \frac{d \sigma_{c}}{d x}
$$

Substitution of (1) into (2) leads to, 


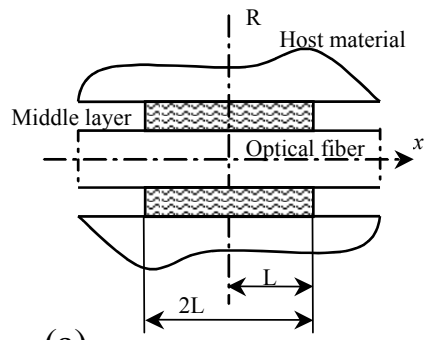

(a)
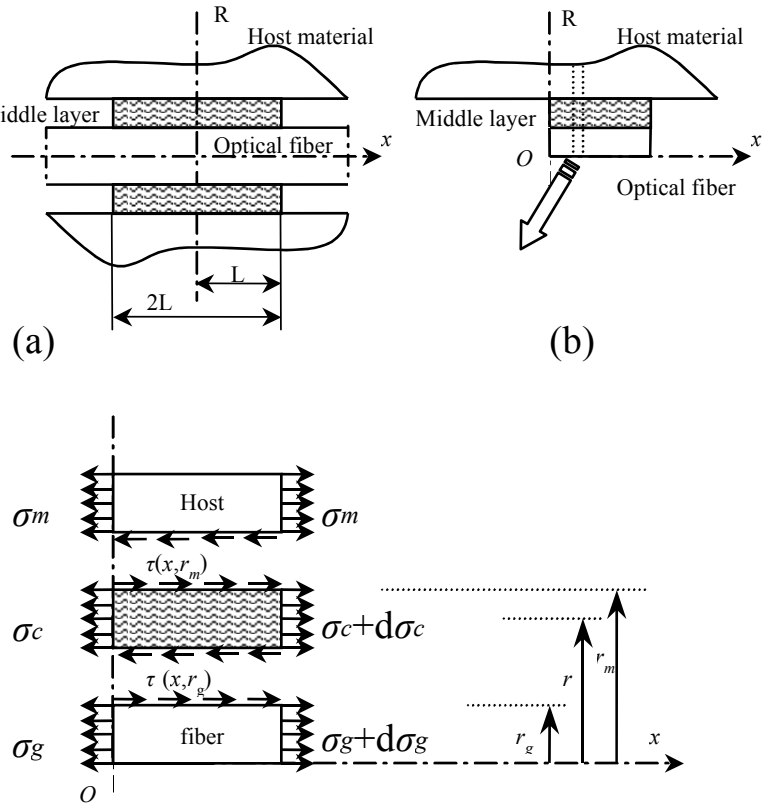

(c)

Fig. 2. Coordinate system and free body diagram of symmetrical section of optical fiber: (a) Optical fiber of gauge length $2 L$; (b) One quarter of the fiber; (c) Stress distribution of the fiber and the coating.

$$
\tau(x, r)=-\frac{r_{g}{ }^{2}}{2 r} \frac{d \sigma_{g}}{d x}-\frac{r^{2}-r_{g}^{2}}{2 r} \frac{d \sigma_{c}}{d x}
$$

Since the shear modulus of deformation predominates when it comes to the load transferring between the host material and the fiber, lateral motions perpendicular to the $x$ coordinate are of secondary importance for the present study, and the Poisson effect can be neglected. Using the constitutive equation relating stress to strain, $\sigma_{g}=E_{g} \varepsilon_{g}$, where $\varepsilon_{g}=\frac{d u}{d x}$ is the axial strain along the fiber and $u \equiv u(x)$ is the displacement, one obtains,

$$
\begin{aligned}
& \tau(x, r)=-\frac{r_{g}{ }^{2}}{2 r} E_{g} \frac{d \varepsilon_{g}}{d x}-\frac{r^{2}-r_{g}{ }^{2}}{2 r} E_{c} \frac{d \varepsilon_{c}}{d x} \\
& =-\frac{E_{g} r_{g}{ }^{2}}{2 r}\left(\frac{d \varepsilon_{g}}{d x}-\frac{r^{2}-r_{g}{ }^{2}}{r_{g}{ }^{2}} \frac{E_{c}}{E_{g}} \frac{d \varepsilon_{c}}{d x}\right)
\end{aligned}
$$

Since the fiber is strained together with the middle layer, the strain gradients are expected to be of the same order,

$$
\frac{d \varepsilon_{g}}{d x} \cong \frac{d \varepsilon_{c}}{d x}
$$


Thus, the important factor that determines $\tau(x, r)$ in (4) is the ratio of the stiffness between the fiber and the middle layer. The Young's modulus of the optical fiber coatings, in the case of a coated fiber, or the Young's modulus of typical structural epoxies, in the instance of a bare embedded fiber is, as usual, ten percent or less than that of the glass fiber, and $r$ is not much larger than $r_{\mathrm{g}}$ (the middle layer is typically very thin to admit efficient strain transfer between the fiber and the host material), the second part of the right hand side of (4) is , therefore, insignificant compared to the first part,

$$
\frac{r^{2}-r_{g}^{2}}{r_{g}^{2}} \frac{E_{c}}{E_{g}} \frac{d \varepsilon_{c}}{d x} \cong o\left(\frac{d \varepsilon_{g}}{d x}\right)
$$

Substituting (5) and (6) into (4) results in,

$$
\tau(x, r)=-\frac{r_{g}{ }^{2}}{2 r} \frac{d \sigma_{g}}{d x}=-\frac{r_{g}{ }^{2}}{2 r} E_{g} \frac{d \varepsilon_{g}}{d x}
$$

The shear stress term in (7) is determined by using Hooke's law as follows,

$$
\tau(x, r)=G_{c} \gamma(x, r)=G_{c}\left(\frac{\partial u}{\partial r}+\frac{\partial w}{\partial x}\right) \cong G_{c} \frac{\partial u}{\partial r}
$$

where $u \equiv u(x)$ and $w \equiv w(x)$ are the axial and radial displacements in the middle layer, respectively. Substituting (8) into (7) and integrating the resulting expression over $r_{\mathrm{g}}$ from the fiber and middle layer interface to the middle layer and host material interface radius $r_{\mathrm{m}}$ gives,

$$
\int_{r_{g}}^{r_{m}} G_{c} \frac{\partial u}{\partial r} d r=\int_{r_{g}}^{r_{m}}\left[-\frac{r_{g}^{2}}{2 r} E_{g} \frac{d \varepsilon_{g}}{d r}\right] d r
$$

The integration result of (9) is,

$$
u_{m}-u_{g}=-\frac{E_{g}}{2 G_{c}} \frac{d \varepsilon_{g}}{d x} r_{g}^{2} \ln \left(\frac{r_{m}}{r_{g}}\right)=-\frac{1}{k^{2}} \frac{d \varepsilon_{g}}{d x}
$$

where $u_{m}$ and $u_{g}$ respectively denote the axial displacement from the $x$ coordinate origin in the host material and fiber. The strain lag parameter, $k$, containing both effects of the geometry and the relative stiffness on the system components, can be written as,

$$
k^{2}=\frac{2 G_{c}}{r_{g}{ }^{2} E_{g} \ln \left(\frac{r_{m}}{r_{g}}\right)}=\frac{1}{(1+\mu) \frac{E_{g}}{E_{c}} r_{g}{ }^{2} \ln \left(\frac{r_{m}}{r_{g}}\right)}
$$

where, $G_{c}$, is the shear modulus of the middle layer. Differentiation of (10) with respect to $x$ yields,

$$
\frac{d^{2} \varepsilon_{g}(x)}{d x^{2}}-k^{2} \varepsilon_{g}(x)=-k^{2} \varepsilon_{m}
$$


The general solution to (12) takes of the following form,

$$
\varepsilon_{g}(x)=c_{1} e^{k x}+c_{2} e^{-k x}+\varepsilon_{m}
$$

where $c_{1}$ and $c_{2}$ represent the integration constants determined by boundary conditions. Since the host material does not contact the fiber beyond the ends of the interface between the fiber and the middle layer, the fiber is assumed to be free from axial stress at both ends. This assumption means that the strain transferred to the fiber is equal to zero at both ends of the OFS, and is given by,

$$
\varepsilon_{g}(L)=\varepsilon_{g}(-L)=0
$$

The boundary conditions established by (14) are identical to the following equations,

$$
\varepsilon_{g}(L)=0, \dot{\sigma}_{g}(0)=0, \dot{\varepsilon}_{g}(0)=0
$$

The relationship (15) states that there is no shear stress in the fiber at the midpoint of the fiber (refer to (1)) due to the symmetric nature of the structure. The boundary conditions established in (14) and (15) will lead to the same solution as (13). Hence, by imposing these boundary conditions on (13), $c_{1}$ and $c_{2}$, are evaluated and obtained as

$$
c_{1}=c_{2}=-\frac{\varepsilon_{m}}{2 \cosh (k L)}
$$

Thus, the final solution to (13), i.e. the strain distribution along fiber and its relationship to the strain of the host material at a given $x$ coordinate is,

$$
\varepsilon_{g}(x)=\varepsilon_{m}\left(1-\frac{\cosh (k x)}{\cosh (k L)}\right)
$$

Eq.(17) is the governing equation that describes the strain distribution along the fiber. The effects of the Young's moduli of the fiber and the middle layer, as well as the effects of the radii of the fiber and the middle layer, on the strain transfer, are all included in the strain lag parameter $k$ defined in (11).

Fig.3 shows the strain transfer rate along an optical fiber. The mechanical properties of the optical fiber employed in the investigation, are given in Table 2. These properties are the same as those used by Ansari \& Libo(1998) for comparison purposes. The strain difference between the fiber and the host material at a given $x$ coordinate, is determined by the strain lag parameter $k$. Fig. 3 also demonstrates that the strain sensed by the fiber at the midpoint is not equal to the strain in the host material in this instance.

\subsection{Influence of the host material}

Previous research on embedded OFSs have been carried out under the assumption that the strain distribution of the host material is not influenced by the embedding of the OFS and equals to the actual strain of the host material on the outer surface of the interlayer. According to Newton's third law, when strain in the host material causes deformations in 


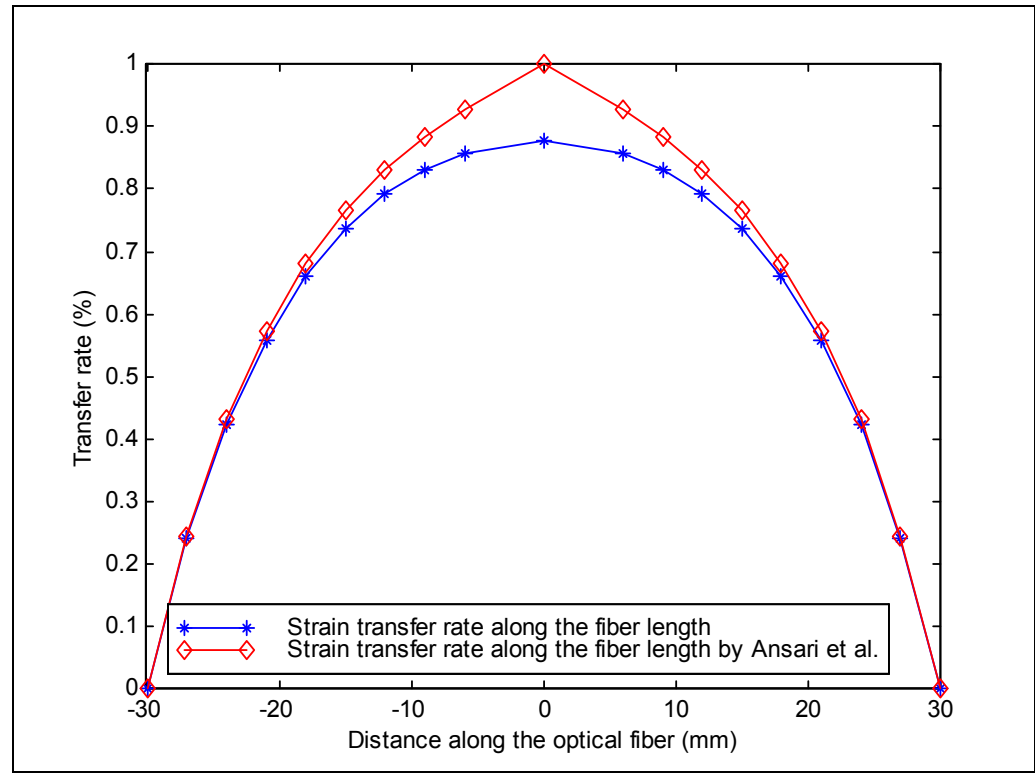

Fig. 3. Distribution of normal strain in fiber along the length

\begin{tabular}{|l|l|l|l|}
\hline \multicolumn{1}{|c|}{$\begin{array}{l}\text { Materials Properties } \\
(1)\end{array}$} & $\begin{array}{c}\text { Symbols } \\
(2)\end{array}$ & \multicolumn{1}{|c|}{$\begin{array}{c}\text { Values } \\
(3)\end{array}$} & \multicolumn{1}{c|}{$\begin{array}{c}\text { Unit } \\
(4)\end{array}$} \\
\hline Young's modulus of the fiber & $E_{\mathrm{g}}$ & $7.2 \times 10^{10}$ & $\mathrm{~Pa}$ \\
\hline Young's modulus of silicon coating & $E_{\mathrm{c}}$ & $2.55 \times 10^{6}$ & $\mathrm{~Pa}$ \\
\hline Poisson's ratio of silicon coating & $\mu$ & 0.48 & - \\
\hline Shear modulus of silicon coating & $\mathrm{Gc}_{\mathrm{c}}$ & $8.5 \times 10^{5}$ & $\mathrm{~Pa}$ \\
\hline Radius of outer boundary of silicon coating & $r_{\mathrm{m}}$ & 102.5 & $\mu \mathrm{m}$ \\
\hline Radius of the fiber & $r_{\mathrm{g}}$ & 62.5 & $\mu \mathrm{m}$ \\
\hline
\end{tabular}

Table 2. Mechanical properties of the optical fiber

the interlayer, the interlayer reacts causing opposite deformations in the host material. Thereby, the strain level of some of the host material surrounding the embedded OFSs is lower than the actual strain of host material. The effects of host material properties in strain transfer are often ignored. However, many experiments and interrelated investigations reveal that this will result in large errors of strain transfer in the calculation especially when the stiffness of host materials is much lower than that of the fiber core.

In the field of short-fiber composites, Cox(1952) first developed the theoretical analysis of load transfer from the matrix to the fiber and this theory is referred to as the shear-lag analysis. The Young's modulus of matrix is considered as an important parameter. Subsequently, Monette et al. (1992), Rosen et al. (1965) and Tsai et al. (1990) deduced different improved strain transfer formulae using different assumptions based on the principle of shear-lag theory. In their formulae, the Young's modulus of matrix was introduced as a variable. All the results indicated that the strain transfer depends on the 
characteristics of the matrix. Monette et al (1992) investigated the strain transfer employing both analytical theory and computer simulation. The conclusions imply that the strain distribution in the fiber is expressed as a function of $E_{f} / E_{m}$, here $E_{f}$ and $E_{m}$ are the Young's modulus of fiber and matrix respectively; the stress transfer efficiency increases as the value of $E_{f} / E_{m}$ increases. In the same paper (Monette et al. 1992), a general theory was outlined based on a shear-lag type approach for a three-phase composite material made of a single fiber plus an interphase region embedded in a soft matrix. The shear strain in the matrix exists from the interphase to a distance chosen far away in the matrix. The computer simulation results infer that the strain transfer is deeply influenced by the ratio, $E_{f} / E_{m}$. Grubb and Li (1994) used Raman spectroscopy to measure the axial stress distribution along a fiber during a quasi-static single fiber pull-out test. They obtained that the shear-lag constant can be expressed as a function of the matrix shear modulus and geometric terms. One of these terms is the effective interfacial radius, $r_{g}$, which is the radius for which the strain in the matrix equals the average matrix strain. Raman measurements indicate that $r_{g}$ is small, only four times the fiber radius. This result is supported by polarizing microscopy. The model of Greszczuk (1969), which assumes a uniform shear within an effective interaction thickness, gives a similar result. Jiang et al. (1998) concluded that the conventional shear-lag theory has many drawbacks. Despite considering the strain transfer from matrix to fiber, the influence of the embedded fiber on the matrix is not taken into account by the shear-lag theory. They developed the theoretical formulae of fiber and matrix strain field distributions employed both shear-lag theory and elastic theory. The significance of the matrix is emphasized sufficiently in their deformation model. They not only considered the strain transfer from the matrix to the fiber, but also considered the influence of the fiber on the behavior of the matrix.

All the research findings indicate that the strain distribution of the host material surrounding the fiber is influenced by the fiber embedding; the character of the host material have important effects on the strain transferring from host material to fiber. Therefore, the mechanical properties of the host material must be taken into account when exploring the strain transferring from host material to fiber.

In our previous investigations(Li et al., 2006; LI et al., 2009), an improved strain transfer model is developed considering the influence of the host material. An additional assumption is that the normal stress and the shear stress are both existent in some range of the host materials and the influencing radius is equal to four times the external radius of the interlayer. The derivation is similar to the simple model and the final solution is in the same form as in Eq.(17) with the only exception that the shear lag parameter, $k$, takes a different expression as follows,

$$
k^{2}=\frac{2}{r_{f}^{2} E_{f}\left\{\frac{1}{G_{c}} \ln \left(\frac{r_{c}}{r_{f}}\right)+\frac{1}{G_{m}}\left[\frac{r_{m}^{2}}{r_{m}^{2}-r_{c}^{2}} \ln \left(\frac{r_{m}}{r_{c}}\right)-\frac{1}{2}\right]\right\}}
$$

\subsection{Average strain transfer rate}

The strain transferred from the host material to an optical fiber varies with the different points along the gauge length of the fiber. Strain transfer rate (STR) $\alpha(x)$ can be defined as 
the ratio of the strain measured by an OFS sensor and the strain actually experienced by the host material at a given point of the fiber (a given $x$ coordinate) as following,

$$
\alpha(x)=\frac{\varepsilon_{g}(x)}{\varepsilon_{m}}=\left(1-\frac{\cosh (k x)}{\cosh (k L)}\right)
$$

The maximum STR $\alpha_{m}(x)$ occurs at the midpoint of the fiber, i.e. at the point where $x$ is equal to zero.

$$
\alpha_{m}(0)=\frac{\varepsilon_{g}(0)}{\varepsilon_{m}}=\left(1-\frac{1}{\cosh (k L)}\right)
$$

Generally, the strain measured by an OFS sensor, is the average strain over the gauge length of the fiber. Average STR (ASTR) can be expressed in the following form,

$$
\bar{\alpha}=\frac{\bar{\varepsilon}_{g}(x)}{\varepsilon_{m}}=\frac{2 \int_{0}^{L} \varepsilon_{g}(x)}{2 L \varepsilon_{m}}=1-\frac{\sinh (k L)}{k L \cosh (k L)}
$$

The ASTR determined by (21) depends on the gauge length and the mechanical properties of the optical fiber, the middle layer and the host material. Therefore, it can be readily employed for correct interpretation of structural strains from the optical fiber measurement values. The results from the developed analytical model can be used as a complement to experiments especially where calibration tests are difficult to perform or where qualitative interpretation of a measurement system is required, for instance, in embedment applications of OFSs.

Fig.4 shows the variation of ASTRs $\bar{\alpha}$ over the fiber length and maximum STRs at the midpoint of the fiber in terms of the gauge length of the optical fiber, and also the experimental values by Ansari and Yuan (1998). It can be seen that the ASTRs and maximum STRs for the optical fiber in our study differs from those derived by Ansari and Yuan (1998), especially for the optical fiber with half-gauge length shorter than $60 \mathrm{~mm}$. When the fiber gauge length increases, the ASTRs and the maximum STRs determined by Ansari and Yuan (1998) are close to the values presented in this paper since $\sinh (k L)$ is nearly equal to $\cosh (k L)$ for a larger fiber half gauge length $L$. It should be emphasized that (21) is a more accurate solution for the strain transfer problem in the concentric cylinder model.

As indicated in Fig.4, the ASTR experimental values by Ansari and Yuan (1998) agree quite well with those evaluated by (21). Although the model has been verified for the simple case, it needs further experiments to validate its generalization. In any case, it facilitates a simple and direct qualitative interpretation of structural strains from measurement values made by OFSs.

Since the STRs at all the points within the fiber gauge length varies and a Bragg grating demands uniform axial deformations to avoid multiple-peak reflection and light spectrum expansion, it is required that an optical fiber be evenly stressed. However, only a short portion of FBG sensor is usually bonded, and this will lead to inadequate strain transfer, i.e. the strain sensed by a FBG sensor is only a fraction of the host material strain. Fig.5 shows 


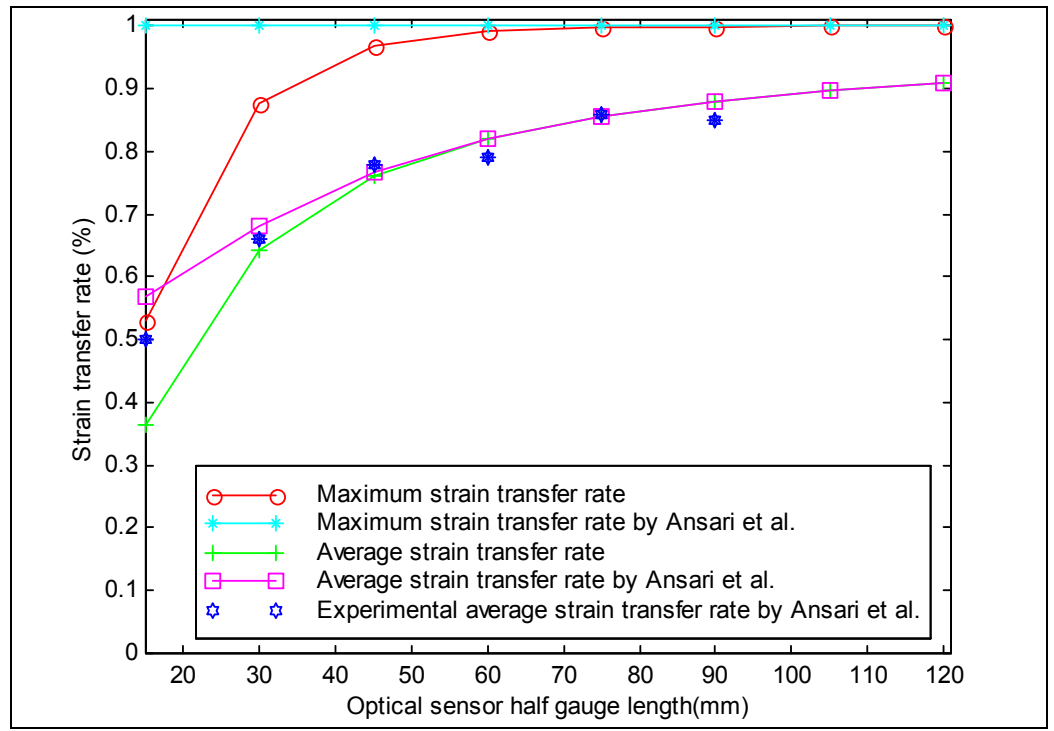

Fig. 4. Comparison of maximum STR and ASTR with experimental data for various gauge length sensors

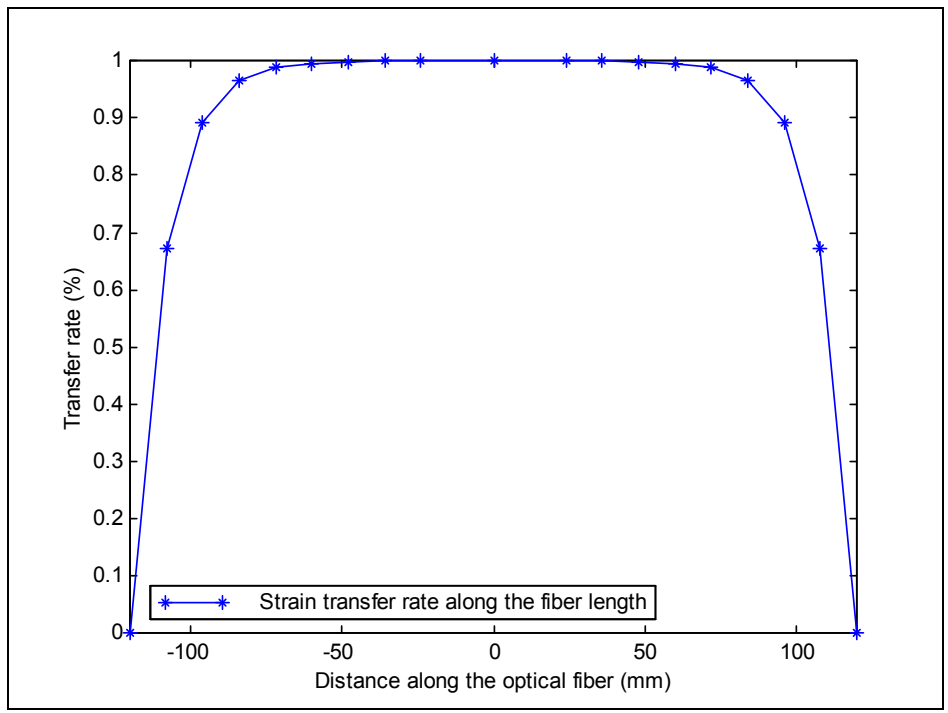

Fig. 5. Strain transfer rate of an $120 \mathrm{~mm}$ gauge length fiber

the variation of ASTRs $\bar{\alpha}$ along the fiber with a gauge length $120 \mathrm{~mm}$. The figure indicates that the STRs near the center of the fiber approach unity. Therefore, an adequate fiber length has to be bonded to ensure the correct measurement of the host structural strain. Critical adherence length (CAL) can be defined as the minimum length to be bonded so that the STRs, at least over the middle half-length of the fiber, are larger than 0.9 , i.e. 


$$
\alpha\left(l_{c} / 2\right) \geq 0.9
$$

Substituting (19) into (22) gives,

$$
\frac{\varepsilon_{g}\left(l_{c} / 2\right)}{\varepsilon_{m}}=1-\frac{\cosh \left(k l_{c} / 2\right)}{\cosh \left(k l_{c}\right)} \geq 0.9
$$

The solution of (23) is,

$$
\mathrm{CAL}=l_{\mathrm{c}}=9.24 / k
$$

where $l_{\mathrm{c}}$ is only computed over the half gauge length. Critical adherence length means that an OFS can be bonded over a longer portion, and the effective gauge length is located in the middle. For the case of the OFS shown in Table 2, its CAL is $99.78 \mathrm{~mm}$. This implies that the STRs along the middle half-length of the fiber are larger than 0.9 as long as the minimum bonded fiber length is beyond $99.78 \mathrm{~mm}$. CAL indicates that we can bond an FBG over a longer length, for instance $80 \mathrm{~mm}$, to locate the FBG just in the middle of the adhered length for efficient strain transferring.

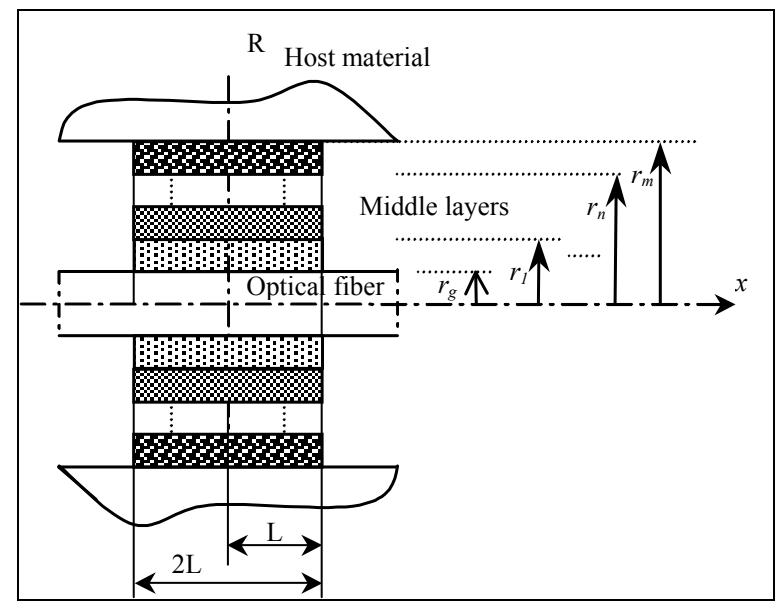

Fig. 6. Cross section of a multi-layer adhered fiber

\subsection{Strain transferring for a multi-layered concentric model}

In many cases, there are several middle layers between the fiber and host material, for instance, the fiber is first coated with an adhesive that solidifies quickly, and then bonded to the structure by an epoxy that solidifies slowly to ensure a uniform stress distribution. One typical case is to bond the fiber to a structure by directly applying adhesives on the fiber coating, and the coating and the adhesive form two separate layers between the fiber and host material. In some sensing applications, specialized coatings are required to enhance an optical fiber's measurement sensitivity and to accommodate the host structure. The OFSs maybe coated in this way with two different layers of coatings to employ their advantages of mechanical properties. 
Fig.6 shows a multiple layer model available for the strain transfer analysis. Additionally, $r_{\mathrm{i}}$ is the outer radius of the ith layer $(i=1 \sim n), r_{\mathrm{g}}$ is the outer radius of the fiber layer and $r_{\mathrm{m}}$ is the inner radius of the host materials (i.e. the outer radius of the $(n+1)$ th layer). $G_{i}$ is the shear modulus of the ith layer $(i=2 \sim n)$, and $G_{c}$ is the shear modulus of the 1st layer.

Equation (9) can be rewritten as,

$$
\int_{r_{g}}^{r_{m}}\left[\frac{d x}{d r}\right] d r=\int_{r_{g}}^{r_{m}}\left[-\frac{1}{G_{c}} \frac{r_{g}{ }^{2}}{2 r} E_{g} \frac{d \varepsilon_{g}}{d x}\right] d r
$$

The resulting expression is integrated, over $r_{\mathrm{g}}$, from the fiber and first layer interface, to the outmost layer and host material interface radius $r_{\mathrm{m}}$ through all the middle layers as follows,

$$
\left(\int_{r_{g}}^{r_{1}}+\int_{r_{1}}^{r_{2}}+\ldots+\int_{r_{n}}^{r_{m}}\left[\frac{d x}{d r}\right]\right) d r=\left(\int_{r_{g}}^{r_{1}}+\int_{r_{1}}^{r_{2}}+\ldots+\int_{r_{n}}^{r_{m}}\left[-\frac{1}{G_{c}} \frac{r_{g}^{2}}{2 r} E_{g} \frac{d \varepsilon_{g}}{d x}\right] d r\right.
$$

The integration result of (25) is given by,

$$
u_{m}-u_{g}=-\frac{1}{k_{m}{ }^{2}} \frac{d \varepsilon_{g}}{d x}=-r_{g}{ }^{2} \frac{E_{g}}{2}\left\{\sum_{i=2}^{n} \frac{1}{G_{i}} \ln \left(\frac{r_{i}}{r_{i-1}}\right)+\frac{1}{G_{c}} \ln \left(\frac{r_{1}}{r_{g}}\right)\right\} \frac{d \varepsilon_{g}}{d x}
$$

where $u_{\mathrm{m}}$ and $u_{\mathrm{g}}$ denotes the axial displacement from the $x$ coordinate origin in the host material and fiber, respectively.

$$
k_{m}{ }^{2}=\frac{2}{r_{g}{ }^{2} E_{g}\left\{\sum_{i=2}^{n} \frac{1}{G_{i}} \ln \left(\frac{r_{i}}{r_{i-1}}\right)+\frac{1}{G_{c}} \ln \left(\frac{r_{1}}{r_{g}}\right)\right\}}
$$

The strain lag parameter $k_{m}$, similar to the formerly discussed case, is determined by Young's moduli of the fiber and the middle layers, and the diameters of the fiber and the middle layers.

Thus, (19) and (28) can be directly used to compute the strain transfer rate for a fiber embedded in host material with multiple layers. Consequently, the critical adherence length and the average strain transfer rate within a gauge length for an optical fiber sensor can be computed by (19) and (28).

\subsection{Test validation}

The experiment is conducted on a universal material testing machine to study the strain transmission characteristics of an FBG sensor on different material sheets through a comparison of the strain values measured with the bare FBG and the electric strain gauge. A steel plate and a plexiglass plate are used in the experiments. Both plates are in rectangular shapes with uniform thickness. Two bare FBG sensors were bonded directly on one side of the plate with the two-component epoxy resin. One is located in the middle of the specimen and the other is located near the end of the specimen. Two electric strain gauges are fixed at the same positions on the other side of the specimen. The experimental specimen is shown in Figs. 7 and 8. 


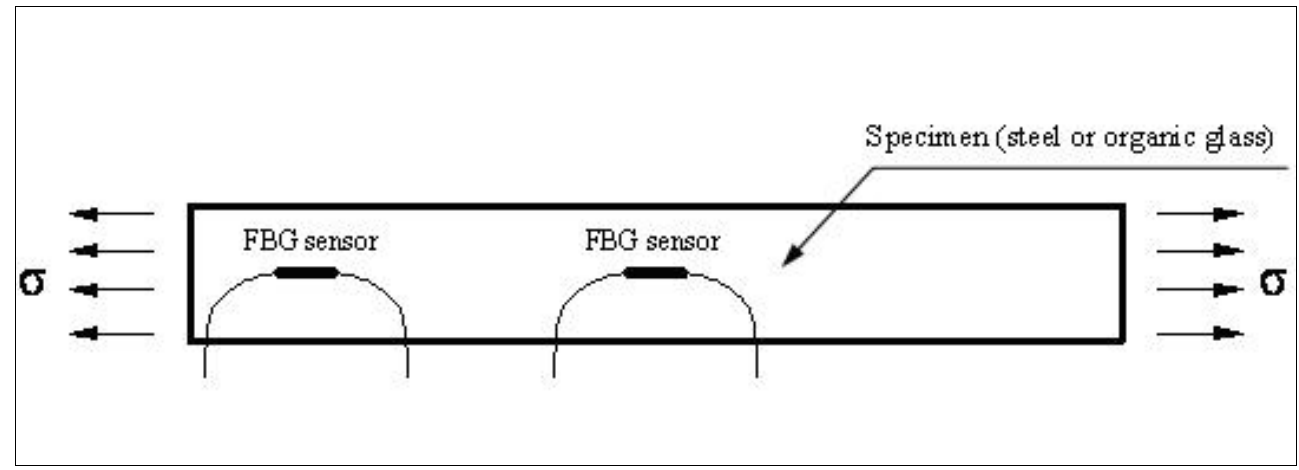

Fig. 7. The plan figure of the FBG sensors distribution.

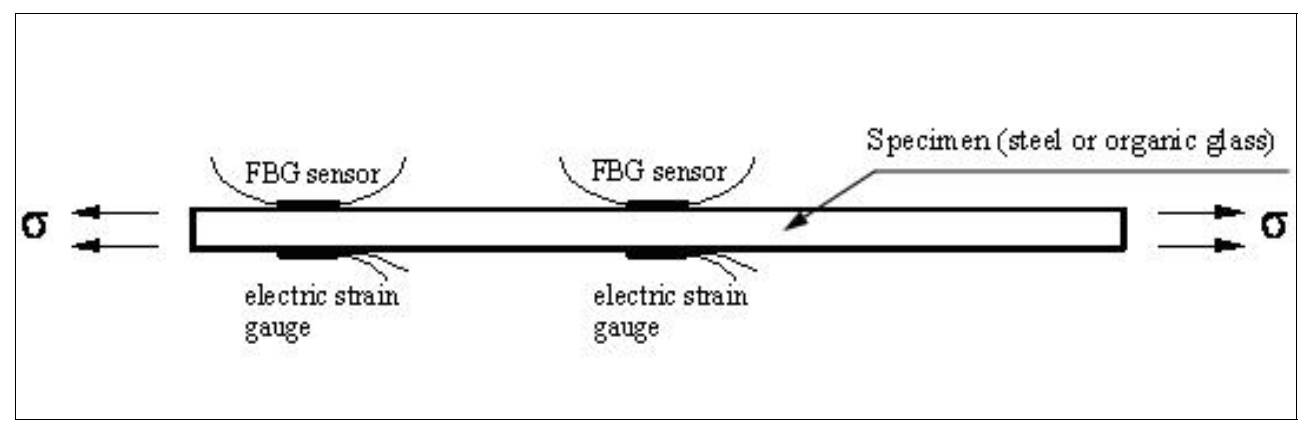

Fig. 8. The profile chart of sensor distribution.

Tensile experiments were carried out on the specimens. The load is controlled in the linear elastic range of the specimens. Therefore, each point of the specimens is under the same strain presumably from the point of view of elastic behaviour. The strain measured by the strain gauge is the actual strain of the plate. The ratio of the strain sensed by the FBG sensor to the strain measured by the strain gauge is considered to represent the strain transfer rate from the host material to the fiber core. As shown in Fig. 9, the steel plates is loaded step by step and continuously from $0 \mu \varepsilon$ to $250 \mu \varepsilon$, then unloaded to $0 \mu \varepsilon$. It is seen that the linearity of strain with the wavelength is very good, and that the coefficient of linear correlation is larger than 0.999. As shown in Fig. 10, the plexiglass sheet is loaded step by step and continuously from $0 \mu$ sto $500 \mu \varepsilon$, and the coefficient of linear correlation is larger than 0.999 as well.

Based on the theory developed in this chapter, the average strain transfer ratios in steel and plexiglass as shown in Eq.(21) are 0.82330 and 0.7892 respectively. The ratio of average strain transfer with two different host materials is 0.9586 . The experiment values can be calculated using test data. The experiments were repeated three times with three specimens and the data are listed in Table 3. All differences between the test and the theoretical values are less than $5 \%$. Thus, the theoretical model is verified and the model is suitable for the analysis of fiber optic sensor strain transfer with different shear modulus of host materials. 


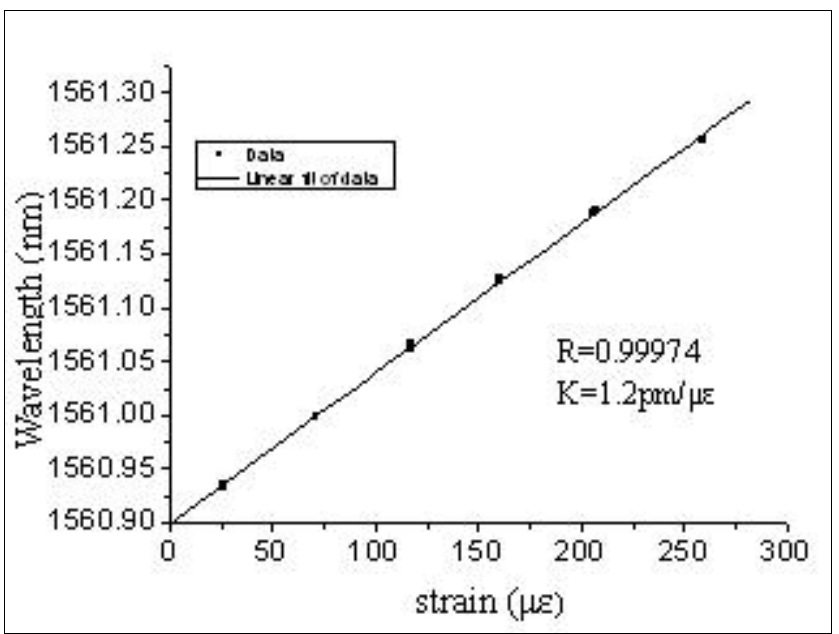

Fig. 9. the relationship between FBG wavelength and strain on the steel plate.

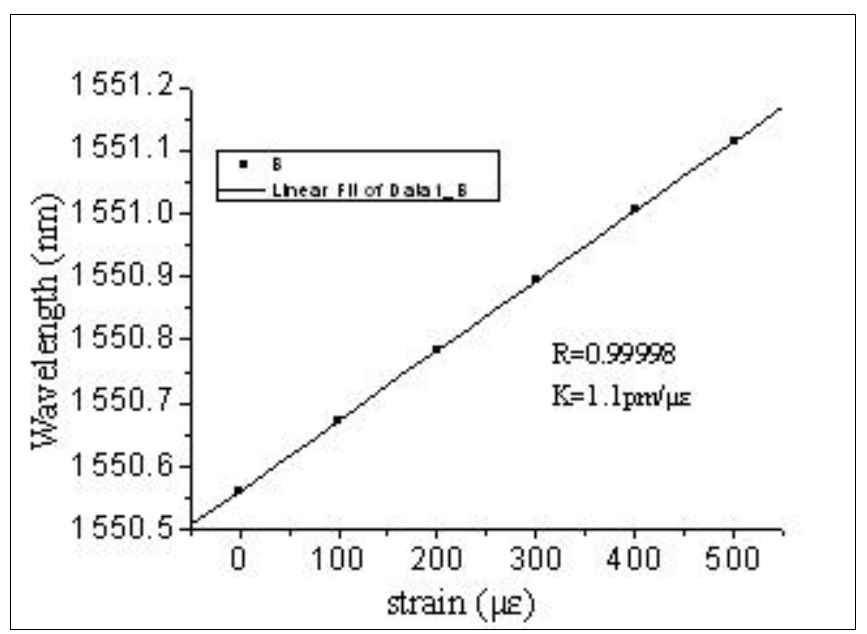

Fig. 10. the relationship between FBG wavelength and strain on the plexiglass plate.

\begin{tabular}{|c|c|c|c|}
\hline Average strain transfer rate & First specimen & Second specimen & Third specimen \\
\hline Experiment values $\left(\mathrm{c}_{1}\right)$ & 0.9602 & 0.9596 & 0.9543 \\
\hline Theoretical values $\left(\mathrm{c}_{2}\right)$ & 0.9586 & 0.9586 & 0.9586 \\
\hline Error $\left(\left(\mathrm{c}_{1}-\mathrm{c}_{2}\right) / \mathrm{c}_{2}\right)$ & $0.2 \%$ & $0.1 \%$ & $0.5 \%$ \\
\hline
\end{tabular}

Table 3. Average strain transfer rates of bonded fiber Bragg grating sensors. 
When the axis of the optical fiber has an angle with the direction of the applied principal strain/ stress, there would be a different strain/stress transfer rate existence because of the interlayer between the fiber optic sensor and the surrounding matrix. Li et al.(2007) discussed this aspect. Jiang \& Peters (2008) developed a shear-lag model for unidirectional multi-layered structures whose constituents vary throughout the cross section through the extension of an existing optimal shear-lag model suitable for two-dimensional planar structures and discussed solution algorithms for a variety of boundary conditions.

\section{Strain transferring mechanism for surface-bonded OFSs}

Apart from embedded FBG sensors, bonded FBG sensors are used more frequently. Because of the asymmetry of the bonded sensors system, the theoretical analysis is difficult. The research methods concentrated on computer simulation with finite element model (FEM) mostly. Betz et al. (2003) studied strain transferring of patch bonded FBG sensors with finite element analysis and experiments. The fiber is first placed on a backing patch and this patch is simply glued to the surface of a structure. The thickness and the modulus of the backing material are varied in the model. When the structure is strained $0.3 \%$ parallel to the direction of the fiber axis, the strain level in the fiber core is found to vary between $0.26 \% \sim 0.28 \%$. It is concluded that the structural strain has not been completely transferred due to the fiber due to the presence of the backing patch, and that the degree to which the strain is transferred depends on the thickness and Young's modulus of the backing. Lin et al., (2005) investigated three packaging methods of FBG sensors and found that strain transmission rates decrease with the increase in thickness of the steel tube. Their experiments show, however, that the thickness and Young's modulus of the glues have little influence on the strain transmission.

Li et al. (2008) and Zhou et al. (2010) found also that the strain transmission loss is small when the substrate is thick and stiff as compared to the bonding layer and the FBG. However, it becomes large when the substrate is thin and made by low modulus materials. Wan et al. (2008) studied strain transfer with stiff adhesive case with a 3D-FEM for a surface-mounted strain sensor and verified it by experiments. They found that the bond length and the bottom thickness are dominant factors besides side width and top thickness. Jahani \& Nobari (2008) demonstrated that both Young's and shear moduli of adhesive are frequency dependent in dynamic testing because adhesive materials show viscoelastic behaviour.

\section{Conclusion}

The strain transfer mechanism from host material to the fiber core can be described as follows: as the host material generates strain under the external load, the non-uniform deformation makes the shear strain appear in the interface between the host material and interlayer, and the shear strain is transferred to the interior of the interlayer to an extent which depends on the effective bonding of the interface. The interlayer generates axial strain at the same time. The axial strain in the interlayer is transferred to the fiber core through the shear strain in the interface between the interlayer and fiber core. The fiber core generates axial strain and senses the strain of the host material. In the course of strain 
transferring from host material to fiber core, a portion of strain is absorbed by the interlayer; the fiber core senses a partial strain of the host material only. Theoretical studies and experiments found that the structural strain may not be completely transferred due to the fiber core due to the presence of the coatings or packaging (middle layer). The strain transfer rate depends not only on geometry and mechanical properties of the middle layer or the material between the fiber and host surface, but on the Young's modulus of the host material. Naturally, packaging methods have significant influences on the strain transfer rate.

In practical applications, primitive protective coatings by fiber manufactures have to be stripped off for sufficient strain transfer rate, or at least its low Young's modulus has to be considered in experiments. Moreover, the aging problem of the adhesive should be considered since the service life of most structures to be measured is generally designed for 50 years or longer and adhesive must be adequate.

\section{Acknowledgment}

This research work was supported by the Science Fund for Creative Research Groups of the National Natural Science Foundation of China (Grant No. 51121005).

\section{References}

Ansari, F., \& Libo, Y. (1998). Mechanics of Bond and Interface Shear Transfer in Optical Fiber Sensors. Journal of Engineering Mechanics,Vol.124, No.4,pp.385-394,ISSN 07339399.

Baldwin, C.;Salter, T.;Niemczuk, J.;Chen, P., \& Kiddy, J. (2002). Structural monitoring of composite marine piles using multiplexed fiber Bragg grating sensors: In-field applications. Smart systems for bridges, structures, and highways,Vol.4696,pp.82,ISSN 0819444448.

Betz, D. C.;Thursby, G.;Culshaw, B., \& Staszewski, W. J. (2003). Acousto-ultrasonic sensing using fiber Bragg gratings. Smart Materials \& Structures,Vol.12, No.1,pp.122128,ISSN 0964-1726.

Cox, H. (1952). The Elasticity and Strength of Paper and other Fibrous Materials. British Journal of Applied Physics,Vol.3, No.MAR,pp.72-79,ISSN 0022-3727.

Feced, R.;Roe-Edwards, M.;Kanellopoulos, S.;Taylor, N., \& Handerek, V. (1997). Mechanical strength degradation of UV exposed optical fibres. Electronics Letters,Vol.33, No.2,pp.157-159,ISSN 0013-5194.

Fernando, G.;Webb, D., \& Ferdinand, P. (2002). OPTICAL-FIBRE SENSORS. MRS Bulletin,Vol.27, No.5,pp.359-361,ISSN 0883-7694.

Friebele, E.;Askins, C.;Bosse, A.;Kersey, A.;Patrick, H.;Pogue, W., et al. (1999). Optical fiber sensors for spacecraft applications. Smart Materials and Structures,Vol.8, No.6,pp.813-838,ISSN 0964-1726.

Galiotis, C.;Young, R.;Yeung, P., \& Batchelder, D. (1984). Study of model polydiacetylene/epoxy composites. Part 1- the axial strain in the fibre. Journal of Materials Science,Vol.19, No.11,pp.3640-3648,ISSN 0022-2461.

Greszczuk, L. Theoretical Studies of the Mechanics of the Fiber-Matrix Interface in Composites.ISSN 0091-3286. 
Grubb, T., \& Li, T. (1994). Single-fiber polymer composites: Part I Interfacial shear strength and stress distribution in the pull-out test. J. Mater. Sci,Vol.29,pp.189".C202,ISSN.

Jahani, K., \& Nobari, A. (2008). Identification of dynamic (Youngs and shear) moduli of a structural adhesive using modal based direct model updating method. Experimental Mechanics,Vol.48, No.5,pp.599-611,ISSN 0014-4851.

Jiang, G., \& Peters, K. (2008). A shear-lag model for three-dimensional, unidirectional multilayered structures. International Journal of Solids and Structures,Vol.45, No.1415,pp.4049-4067,ISSN 0020-7683.

Jiang, Z.;Lian, J.;Yang, D., \& Dong, S. (1998). An analytical study of the influence of thermal residual stresses on the elastic and yield behaviors of short fiber-reinforced metal matrix composites. Materials Science and Engineering: A,Vol.248, No.1-2,pp.256275,ISSN 0921-5093.

Li, D. S.;Li Sr, H.;Ren, L., \& Song, G. (2006). Strain transferring analysis of fiber Bragg grating sensors. Optical Engineering,Vol.45, No.2,pp.4402,ISSN 0091-3286.

LI, H. N.;Zhou, G. D.;Ren, L., \& LI, D. S. (2009). Strain Transfer Coefficient Analyses for Embedded Fiber Bragg Grating Sensors in Different Host Materials. Journal of Engineering Mechanics,Vol.135, No.12,pp.1343-1353,ISSN 0733-9399.

Li, H. N.;Zhou, G. D.;Ren, L., \& Li, D. S. (2007). Strain transfer analysis of embedded fiber Bragg grating sensor under nonaxial stress. Optical Engineering, Vol.46 ,pp.054402,ISSN 0091-3286.

Li, Q.;Li, G.;Wang, G.;Ansari, F., \& Liu, Q. (2002). Elasto-Plastic Bonding of Embedded Optical Fiber Sensors in Concrete. Journal of Engineering Mechanics,Vol.128, No.4,pp.471-478,ISSN 0733-9399.

Li, W. Y.;Cheng, C. C., \& Lo, Y. L. (2008). Investigation of strain transmission of surfacebonded FBGs used as strain sensors. Sensors \& Actuators: A. Physical,ISSN 00913286.

Lin, Y. B.;Chang, K. C.;Chern, J. C., \& Wang, L. A. (2005). Packaging methods of fiber-Bragg grating sensors in civil structure applications. Sensors Journal, IEEE,Vol.5, No.3,pp.419-424,ISSN 1530-437X.

Measures, R. M. (2001). Structural monitoring with fiber optic technology ISBN 0124874304: Academic.

Monette, L.;Anderson, M.;Ling, S., \& Grest, G. (1992). Effect of modulus and cohesive energy on critical fibre length in fibre-reinforced composites. Journal of Materials Science,Vol.27, No.16,pp.4393-4405,ISSN 0022-2461.

Pak, Y. (1992). Longitudinal Shear Transfer in Fiber Optic Sensors. Smart Materials and Structures(UK), No.1,pp.57-62,ISSN 0964-1726.

Rosen, B. W. (1965). Mechanics of composite strengthening. Fiber composite materials,Vol.2,pp.37-75,ISSN 0091-3286.

Thyagarajan, K., \& Ghatak, A. K. (2007). Fiber optic essentials ISBN 0470097426: Wiley-IEEE Press.

Tsai, H. C.;Arocho, A. M., \& Gause, L. W. (1990). Prediction of fiber-matrix interphase properties and their influence on interface stress, displacement and fracture toughness of composite material. Materials Science and Engineering: A,Vol.126, No.12,pp.295-304,ISSN 0921-5093.

Udd, E. (1995). Fiber optic smart structures ISBN 0471554480: Wiley-Interscience. 
Varelas, D.;Limberger, H., \& Salathe, R. (1997). Enhanced mechanical performance of singlemode optical fibres irradiated by a CW UV laser. Electronics Letters,Vol.33, No.8,pp.704-705,ISSN 0013-5194.

Wan, K. T.;Leung, C. K. Y., \& Olson, N. G. (2008). Investigation of the strain transfer for surface-attached optical fiber strain sensors. Smart Material Structures,Vol.17, No.3,pp.5037,ISSN 0964-1726.

Zhou, J.;Zhou, Z., \& Zhang, D. (2010). Study on Strain Transfer Characteristics of Fiber Bragg Grating Sensors. Journal of Intelligent Material Systems and Structures,ISSN 1045-389X. 


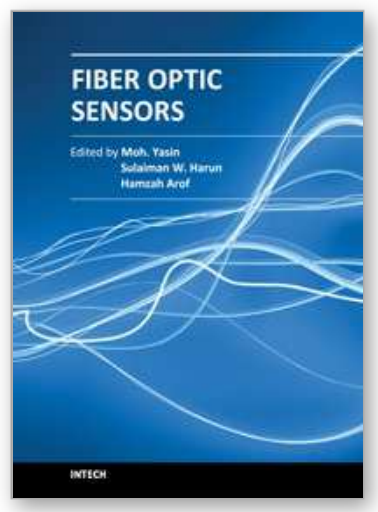

\author{
Fiber Optic Sensors \\ Edited by Dr Moh. Yasin
}

ISBN 978-953-307-922-6

Hard cover, 518 pages

Publisher InTech

Published online 22, February, 2012

Published in print edition February, 2012

This book presents a comprehensive account of recent advances and researches in fiber optic sensor technology. It consists of 21 chapters encompassing the recent progress in the subject, basic principles of various sensor types, their applications in structural health monitoring and the measurement of various physical, chemical and biological parameters. It also highlights the development of fiber optic sensors, their applications by providing various new methods for sensing and systems, and describing recent developments in fiber Bragg grating, tapered optical fiber, polymer optical fiber, long period fiber grating, reflectometry and interefometry based sensors. Edited by three scientists with a wide knowledge of the field and the community, the book brings together leading academics and practitioners in a comprehensive and incisive treatment of the subject. This is an essential reference for researchers working and teaching in optical fiber sensor technology, and for industrial users who need to be aware of current developments and new areas in optical fiber sensor devices.

\title{
How to reference
}

In order to correctly reference this scholarly work, feel free to copy and paste the following:

Dongsheng Li, Liang Ren and Hongnan Li (2012). Mechanical Property and Strain Transferring Mechanism in Optical Fiber Sensors, Fiber Optic Sensors, Dr Moh. Yasin (Ed.), ISBN: 978-953-307-922-6, InTech, Available from: http://www.intechopen.com/books/fiber-optic-sensors/mechanical-property-and-strain-transferringmechanism-in-optical-fiber-sensors

\section{INTECH}

open science | open minds

\section{InTech Europe}

University Campus STeP Ri

Slavka Krautzeka 83/A

51000 Rijeka, Croatia

Phone: +385 (51) 770447

Fax: +385 (51) 686166

www.intechopen.com

\section{InTech China}

Unit 405, Office Block, Hotel Equatorial Shanghai

No.65, Yan An Road (West), Shanghai, 200040, China

中国上海市延安西路 65 号上海国际贵都大饭店办公楼 405 单元

Phone: +86-21-62489820

Fax: $+86-21-62489821$ 
(C) 2012 The Author(s). Licensee IntechOpen. This is an open access article distributed under the terms of the Creative Commons Attribution 3.0 License, which permits unrestricted use, distribution, and reproduction in any medium, provided the original work is properly cited. 